\title{
Enhanced nutritional programme: an innovative approach to controlling plant diseases in the tropics
}

\begin{abstract}
Mineral nutrients are essential for the normal growth and development of both plants and microorganisms and play a crucial role in plant-pathogen interactions, which could be beneficial or detrimental to plant health. Excessive nutrient uptake causes toxicity in plants, so meeting optimal nutrient requirements is very important in the prevention of plant nutrient-deficiency symptoms. In most cases, mineral nutrients represent the primary lines of plant defense against plant pathogens and are directly affected by the plant host, pathogens, and environmental factors. In general, mineral nutrients may affect disease resistance through induced defenses, including by the production of toxins, metabolites, and lignin. The application of an enhanced nutritional programme (ENP) to minimize the deleterious effects of phytopathogens has become a hot topic of discussion around the world. Furthermore, various reports from several countries have shown that the application of mineral nutrients such as calcium $(\mathrm{Ca})$, copper $(\mathrm{Cu})$, and zinc $(\mathrm{Zn})$ in a disease-control programme could enhance crop production and quality, suppress various plant diseases, and promote plant growth. Thus, the aim of this chapter is to discuss conventional disease control methods in the tropics with special reference to major diseases in rice, oil palm, and citrus, as well as limitations on their control. Moreover, the potential application of an ENP as part of a disease control programme in plantation crops in Malaysia is also discussed.
\end{abstract}

Keyword: Basal stem rot; Enhanced nutritional programme; Huanglongbing disease; Plant disease; Tropics 